\title{
Assessment of the Validity of a Physical Activity Questionnaire for Vietnamese Children Using an Accelerometer
}

\author{
Nozomi Watanabe ${ }^{1}$, Keiko Nakamura ${ }^{1^{*}}$, Hoang Thuy Linh Nguyen ${ }^{1,2,3}$, Kaoruko Seino ${ }^{1}$ and Thang Vo Van ${ }^{2,3}$ \\ ${ }^{1}$ Department of Global Health Entrepreneurship, Tokyo Medical and Dental University, Japan \\ ${ }^{2}$ Faculty of Public Health, Hue University of Medicine and Pharmacy, Hue University, Viet Nam \\ ${ }^{3}$ Institute for Community Health Research, Hue University of Medicine and Pharmacy, Hue University, Viet Nam
}

\begin{abstract}
Background: As physical inactivity is becoming a major cause of health issues around the world, not only in adults but also among children, preventions are required especially in low-and middle- income countries. This study was performed to validate a physical activity questionnaire and assess physical activity of 11-year-old children using the questionnaire and a triaxial accelerometer in Hue City, Vietnam.

Methods: A physical activity questionnaire developed by the authors was administered in 30 participants between January and February 2018. Anthropometric measures and 3-day activity monitored using an accelerometer were also obtained. Differences in physical characteristics and physical activity records according to gender and body mass index (BMI), and the relationship between amount of physical activity determined from the questionnaire and accelerometer data were examined.
\end{abstract}

Results: Data for 12 participants were excluded from the analysis due to various types of error. Thirteen of the remaining 18 participants (72\%) achieved more than 60 minutes of moderate-to-vigorous physical activity (MVPA) for all three recording days. Male participants showed higher counts than females in exercise, steps, MVPA and vigorous physical activity (VPA), respectively. Significant relationships were found between accelerometer' records and responses to questions about sedentary behavior, such as time spent playing computer games but not to questions related to their sports activities.

Conclusion: Accelerometers could be used to monitor physical activity among 11-year-old Vietnamese children with support from school teachers or guardians. Accelerometer records were correlated with questions regarding sedentary activities but not questions on sports activities. The results suggested the importance of including questions related to sedentary behavior in physical activity questionnaires. Further studies are needed with larger sample sizes to assess the validity of the questionnaire.

\section{Introduction}

Almost 75\% of non-communicable disease (NCD)-related deaths worldwide occur in low-and middle-income countries [1]. Physical activity is an effective means of preventing NCDs such as heart disease, stroke and diabetes, as well as improving quality of life and overall well-being. However, inadequate daily physical activity is becoming a major health issue in many countries with the World Health Organization (WHO) reporting that $23 \%$ of the world's adult population does not participate in adequate amount of daily physical activity. The level of physical inactivity tends to increase with economic development, changes in transportation patterns, and urbanization [2]. There is a great deal of concern regarding lack of physical activity not only among adults but also among children. In $2016,78 \%$ of boys and $84 \%$ of girls between 11 and 17 years old were reported to lack daily physical activity worldwide [1].

In Vietnam, particularly in urbanized areas, school children have begun to spend large parts of their day studying for competitive exams to enroll in higher education from as early as junior high school. In addition to stress due to pressure from teachers and parents, lack of physical activity due to intensive studying may also have negative effects on their mental health and quality of life [3]. However, there have been no studies regarding the level of physical activity among children in Hue City, the capital of Thua Thien Hue Province located in central Vietnam. The population of Thua Thien Hue Province was $1,154,310$, which consists $12 \%$ of the total population of Vietnam [4, 5]. With rapid economic development and urbanization of cities, it is important to promote physical activity from an early stage of life to prevent future risk of NCDs in the Vietnamese population.

\section{Methods} org/10.15344/2455-7498/2019/149

\section{Publication History:}

Received: February 28, 2019

Accepted: March 16, 2019

Published: March 18, 2019

\section{Keywords:}

Child, Exercise, Fitness trackers, Surveys and Questionnaires

\section{Abbreviations:}

BMI: Body mass index

CDC: Centers for Disease Control and Prevention

EX: Exercise

METs: Metabolic Equivalents MVPA: Moderate to vigorous physical activity

NCD: Non-communicable diseases SPA:Sedentary physical activity VPA: Vigorous physical activity WHO: World Health Organization

Questionnaires are often used to assess amount of physical activity in epidemiological studies due to the low cost of implementation and such surveys often require less than 1 hour for each participant [6]. However, the validity of such questionnaires for use in Vietnamese children has not been documented. There is often concern regarding the poor relationships between actual activity patterns and questionnaire answers, and variations may appear in each individual because the questionnaire is based on participants' subjective responses. Therefore, there is a trend toward using objective measures such as the use of accelerometers, to quantify physical activity.

This study was performed to assess the feasibility and applicability of a triaxial accelerometer to quantify physical activity in children in Vietnam. We also examined the validity of a self-reported questionnaire on physical activity in children in Vietnam by comparison with the results obtained with a triaxial accelerometer.

Hue City consists of $706,732 \mathrm{~km}^{2}$ area located in central part of Vietnam with approximate population of 350,000 (2015). The annual

"Corresponding Author: Prof. Keiko Nakamura, Department of Global Health Entrepreneurship, Tokyo Medical and Dental University, Yushima 1-5-45, Bunkyoku, Tokyo 113-8510, Japan; E-mail: nakamura.ith@tmd.ac.jp

Citation: Watanabe N, Nakamura K, Nguyen HTL, Seino K, Van TV (2019) Assessment of the Validity of a Physical Activity Questionnaire for Vietnamese Children Using an Accelerometer . Int J Phys Ther Rehab 5: 149. doi: https://doi.

Copyright: (C) 2019 Watanabe et al. This is an open-access article distributed under the terms of the Creative Commons Attribution License, which permits unrestricted use, distribution, and reproduction in any medium, provided the original author and source are credited. 
Citation: Watanabe N, Nakamura K, Nguyen HTL, Seino K, Van TV (2019) Assessment of the Validity of a Physical Activity Questionnaire for Vietnamese Children Using an Accelerometer . Int J Phys Ther Rehab 5: 149. doi: https://doi.org/10.15344/2455-7498/2019/149

Page 2 of 4

per capita income of 2018 reached USD 2,750 [7, 8]. Thirty children (14 males and 16 females,) aged 11 years old (as of January 1, 2018) were recruited for this cross-sectional study with data collection from January to February 2018. The participants were volunteers from two different junior high schools in Hue City. Informed consent and informed assent were obtained from all participants and their parents/ guardians prior to enrollment in the study. The study was approved by Tokyo Medical and Dental University Ethics Committee (M2017-211) and Hue University of Medicine and Pharmacy Ethics Committee.

Data were collected from the participants for a total of three week days. On the first day of data collection, a research assistant measured height and weight without footwear and heavy clothing. Participants then moved to another room to answer the questionnaire which was administered orally by a researcher. The questionnaire on physical activity of children was developed using the Vietnamese version of the Global School Health Questionnaire, developed by the WHO and Centers for Disease Control and Prevention (CDC). The questionnaire included six questions related to general physical activity, participation in sports, sedentary behaviors including self-study time and playing computer/video games. At the end of the questionnaire, the participants were instructed how to fit the accelerometer device (Active style pro HJA-750C; Omron Healthcare Co., Ltd., Kyoto, Japan) using a manual. This triaxial accelerometer measuring $52 \times 40 \times 12 \mathrm{~mm}$ and weighing $23 \mathrm{~g}$ detects metabolic equivalents (METs) every $10 \mathrm{~s}$ or 1 -minute epoch and identifies participants' movements as sedentary, walking, or daily activity based on an original algorithm developed by the manufacturer. A 1-minute epoch was used in this study. METs were classified as sedentary physical activity (SPA, $<3 \mathrm{METs}$ ), moderate to vigorous physical activity (MVPA, $\geq 3 \mathrm{METs},<6 \mathrm{METs}$ ), and vigorous physical activity (VPA, $\geq 6 \mathrm{METs}$ ) [9]. Participants were asked to wear the accelerometer throughout the day on the right side of the waist with the attached clip, removing it only when in bed at night or when taking a shower or swimming. Participants were given record sheets and asked to recall and write down daily activities and durations at the end of the day for supplementary information [10]. The accelerometer and record sheets were collected after three full days of recording. Accelerometer data were considered valid only when they contained $>10$ hours of recording time per day for all 3 days [11]. The "Global Recommendations on Physical Activity for Health" issued by the WHO [12], recommends that adolescents should participate in MVPA or VPA at least 3 days per week to prevent various diseases. In this study, participants were considered active when the accelerometer showed more than 60 minutes of MVPA/day.

\section{Statistical analysis}

Statistical analyses were performed using SPSS Version 16.0 (SPSS Inc., Chicago, IL). Body mass index (BMI $\left.\mathrm{kg} / \mathrm{m}^{2}\right)$ was calculated from the height and weight of participants and they were divided into four levels of weight status based on the 2007 WHO reference BMI-forage (boys and girls) at age 11:6 underweight, normal, overweight and obese [13]. Exercise (EX) as a measure of the daily amount of physical activity was calculated by multiplying METs and duration of activity (hours). The means and standard deviations of METs and EX were calculated from the 3-day records. Independent $t$ test, Mann Whitney U test, and Pearson's correlation test were used to assess the differences in physical characteristics and physical activity records among BMI groups and between genders. The Mann Whitney U test [6], Fisher's exact test and Pearson's correlation test were used to compare the relationships between the physical activity questionnaire responses and records from the accelerometer.

\section{Results}

A total of 30 children aged 11 years were enrolled in the study. However, 12 samples were excluded due to device error $(n=2)$, lack of accelerometer record time $(n=9)$ or incomplete questionnaire $(n$ $=1)$. Therefore, further analyses were performed in 18 children $(60 \%)$.

The characteristics of the 18 participants used for analysis are shown in Table 1. There were no significant differences in average height or weight between male and female participants. One participant (6\%) was categorized as "obese," nine (50\%) were categorized as "overweight," and two (11\%) were categorized as "underweight" according to the WHO BMI classification [13].

\begin{tabular}{|l|l|l|l|}
\hline Characteristics & $\begin{array}{l}\text { Total } \\
(\mathrm{n}=18)\end{array}$ & $\begin{array}{l}\text { Male } \\
(\mathrm{n}=7)\end{array}$ & $\begin{array}{l}\text { Female } \\
(\mathrm{n}=11)\end{array}$ \\
\hline Height $(\mathrm{m})$ & $1.45(0.07)$ & $1.41(0.07)$ & $1.46(0.07)$ \\
\hline Weight $(\mathrm{kg})$ & $40.81(9.37)$ & $39.43(9.47)$ & $41.68(9.65)$ \\
\hline BMI $\left(\mathrm{kg} / \mathrm{m}^{2}\right)$ & $19.40(3.75)$ & $19.64(3.72)$ & $19.24(3.95)$ \\
\hline BMI-for-age and gender & \multicolumn{5}{|l}{} \\
\hline$<-2$ SD (Underweight) & 2 & 0 & 2 \\
\hline-2 SD +1SD (Normal) & 6 & 2 & 4 \\
\hline $\begin{array}{l}\text { +1SD +2SD } \\
(\text { Overweight })\end{array}$ & 9 & 4 & 5 \\
\hline$>2 S D($ Obese $)$ & 1 & 1 & 0 \\
\hline
\end{tabular}

Table 1: Characteristics of participants.

Data are presented as means (SD). Weight status is presented as the number of participants.

\section{Triaxial accelerometer measurements}

Thirteen participants (72\%) achieved $>60$ minutes of MVPA for all three recording days, with males showing higher counts than females by $22-81$ points for EX, steps, MVPA, and VPA (Table 2).

Males and females showed different routines of activity, with females tending to increase their physical activity by participating in indoor sports or the use of a bike or by walking to school, while boys spent more time in outdoor sports activities.

There were no significant relationships between BMI and amounts of EX, MVPA, or VPA. All of the overweight and obese participants completed at least 60 minutes of MVPA every day, and six (60\%) of the overweight/obese participants had average EX higher than the average EX for all participants (6.7 EX/day). Seven of the overweight/ obese participants $(70 \%)$ responded that they were willing to lose weight.

\section{Results of the questionnaire and relationships with the accelerometer results}

Six of the 18 participants reported that they were not physically active. Five reported that they had not played sports in the past 7 days, 14 reported they had not played indoor sports in the past 7 days, 10 had not played in any sport teams over the past 12 months, four 
Citation: Watanabe N, Nakamura K, Nguyen HTL, Seino K, Van TV (2019) Assessment of the Validity of a Physical Activity Questionnaire for Vietnamese Children Using an Accelerometer . Int J Phys Ther Rehab 5: 149. doi: https://doi.org/10.15344/2455-7498/2019/149

Page 3 of 4

reported spending more than 2 hours per day in self-study, and eight reported spending more than 1 hour per day to playing computer or video games on weekdays.

Some of the questions showed consistency with the accelerometer records. Children who reported having a low level of physical activity on the questionnaire showed an average of $7-30$ points shorter duration of MVPA on accelerometer records compared to those reporting higher levels of physical activity. This correlation was especially strong for those who reported having spent more time in SPA on the questionnaire. Participants who reported spending $>1$ hour playing computer games or video games per day had 76.9 minutes/day of MVPA, while the value was $104.6 \mathrm{~min} /$ day for those who spent $<1$ hour engaged in such games $(P<0.05)$ (Figure 1 ) The decrease in duration of VPA was seen for children who played computer and video games $\geq 1$ hour (Figure 2). There was significant difference in time spent for VPA between children who played games for $\geq 1$ hour and those who played $<1$ hour $(\mathrm{P}<0.05)$. However, there was little correlation between questions regarding sports activities and amount of physical activity indicated by the accelerometer data.

\begin{tabular}{|l|l|l|l|l|}
\hline & $\begin{array}{l}\text { Total } \\
(\mathrm{n}=18)\end{array}$ & $\begin{array}{l}\text { Male } \\
(\mathrm{n}=7)\end{array}$ & $\begin{array}{l}\text { Female } \\
(\mathrm{n}=11)\end{array}$ & $\begin{array}{l}\text { Male-Female } \\
P \text {-value }\end{array}$ \\
\hline Exercise (EX) & $6.73(2.35)$ & $7.90(2.79)$ & $5.98(1.78)$ & NS \\
\hline Steps (count) & $7110(2760)$ & $8859(3318)$ & $5997(1684)$ & NS \\
\hline MVPA (min) & $92.3(28.6)$ & $103.7(34.4)$ & $85.0(23.1)$ & NS \\
\hline VPA (min) & $7.7(4.2)$ & $10.7(5.0)$ & $5.9(7.7)$ & $<0.05$ \\
\hline
\end{tabular}

Table 2: Summary of accelerometer data.

Data are presented as means (SD) average record for 3 days.

How many days were you physically active in a week?

How many hours did you spend playing sports indoor?

Did you play in any sport teams for the past 12 months?

\author{
How many hours did you spend self studying? \\ How many hours did you spend playing on a computer or \\ video game in a weekday?
}

\section{Discussion}

This was the first study utilizing both subjective and objective measures of daily physical activity determined using an accelerometer and weight status of 11-year-old school children in Hue City, Vietnam. Male participants showed higher levels of physical activity than female participants. Significant relationships were found between accelerometer records and responses to questions regarding sedentary behavior, such as time spent playing computer or video games, but not to questions related to sports activities.

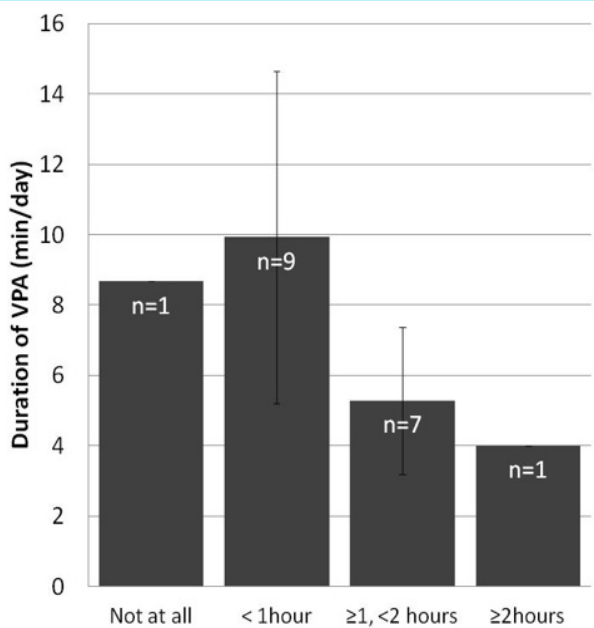

How many hours did you spend playing on a computer or video game in a weekday?

Figure 2: Mean and SD of time spent on vigorous physical activity (VPA) by reports of sedentary activity and physical activity from Vietnamese children.

\section{Neren}

21

\section{.}$$
\text { 2 }
$$

$$
0
$$

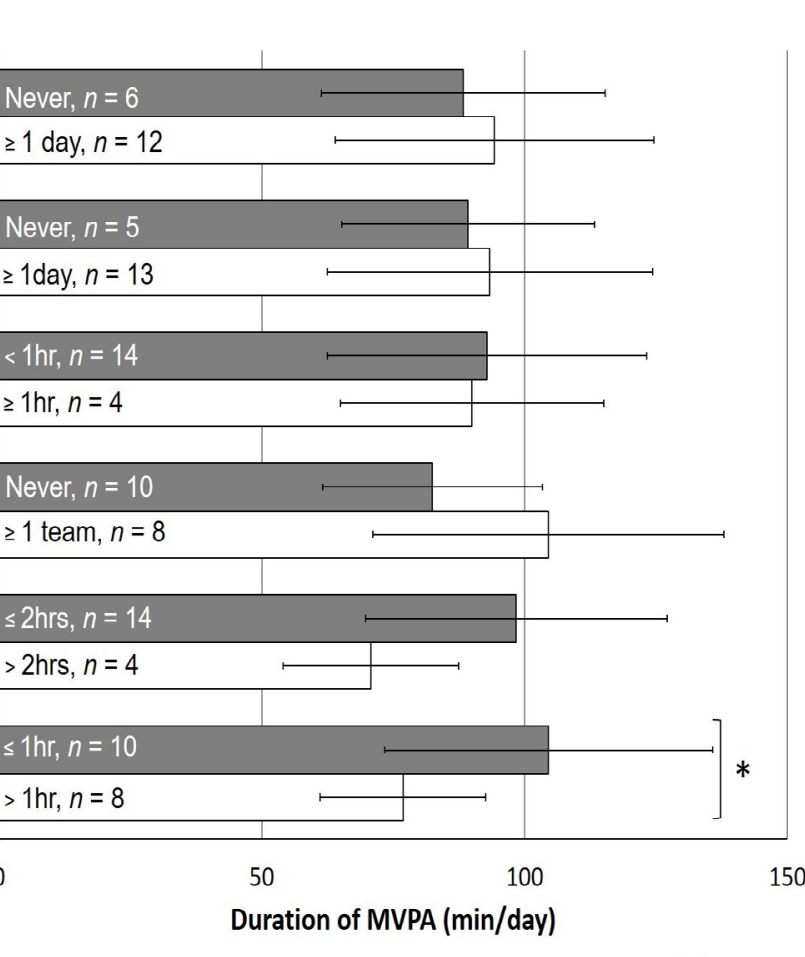

$* P<0.05$

Figure 1: Mean and SD of time spent on moderate to vigorous physical activity (MVPA) by reports of sedentary activity and physical activity from Vietnamese children. 
Citation: Watanabe N, Nakamura K, Nguyen HTL, Seino K, Van TV (2019) Assessment of the Validity of a Physical Activity Questionnaire for Vietnamese Children Using an Accelerometer . Int J Phys Ther Rehab 5: 149. doi: https://doi.org/10.15344/2455-7498/2019/149

Page 4 of 4

Use of accelerometer to evaluate physical activity levels of Vietnamese children

Among the 30 participants originally enrolled in the study, data from 12 cases ( 7 males and 5 females) were not valid due to device error, lack of accelerometer record time, or missing responses in the questionnaire. However, data from $60 \%$ of participants were valid for analysis, which was satisfactory compared to other studies in participants of similar ages [14-16]. The participants received both verbal and written instruction on usage of the accelerometer, and were told specifically when to wear or remove the accelerometer Continuous activity counts of 0 were seen among both successful and unsuccessful participants during the day, which may have corresponded to nap time after lunch, which is a common habit in Hue, or forgetting to reattach the device after changing out of their school uniform. Some participants continued to wear the device at night when in bed. However, some participants followed the instructions carefully and reported precise details on their daily activity record sheets. To maintain good quality datasets, the support of school teachers and parents was required for data collection.

\section{Validity of the questionnaire}

Accelerometer counts showed good correlations with reports of sedentary behavior but not with reports of sports activities. Questions related to sedentary behavior, such as those asking about studying or playing video games may had been easier for the children to understand than those asking about their involvement in sports activities. It is also possible that children participated in physical activity unknowingly as many were found to have been actively moving even during the 5 minute breaks between classes, which they may not have considered as physical activity. It is important to include questions related to sedentary behavior in a physical activity questionnaire, while questions related to sports activities should be translated carefully into the Vietnamese context if they are to be used as a proxy for monitoring children's physical activity.

\section{Activity behavior of Vietnamese children}

According to accelerometer measurements, the majority of participants fulfilled the WHO recommendations for physical activity, i.e., > 60 minutes of MVPA daily with a sufficient amount of VPA. However, more than half of the participants were classified as either overweight or obese. It is possible that these overweight and obese participants engaged more in physical activity only during the period of data collection as half of the overweight or obese participants responded that they felt that they lacked physical activity. Dietary habits should also be taken into consideration in future studies to evaluate the children's nutritional condition. In addition, the Global Recommendations on Physical Activity for Health 2010 may not be applicable for Vietnamese children in terms of preventing excess weight or future risk of NCDs.

\section{Limitations}

This study had some limitations. First, the analysis was performed with a small number of participants and may not be generalizable to the whole population. Second, we did not consider the part of the day that the participants wore the accelerometer as long as they achieved 10 hours of records per day. Therefore, more activity would have been recorded on days on which the children wore the accelerometer for longer times, which would have resulted in longer coverage, thus resulting in a gap in duration of physical activity.

\section{Conclusion}

Accelerometers were applicable for monitoring physical activity level among Vietnamese 11 year-old children, with supports from school teachers and parents/guardians. The accelerometer records showed correlations with some questions regarding sedentary activities, but not with questions regarding sports activities. The observations of the present study suggested the importance of including questions related to sedentary behavior in physical activity questionnaires. Further studies with larger sample sizes are required to assess the validity of this questionnaire.

\section{Funding}

This research was funded by Japanese Society of Promotion of Science (17H02164).

\section{Competing Interests}

The authors declare that they have no competing interests.

\section{References}

1. Sallis JF, Bull F, Guthold R, Heath GW, Inoue S, Kelly P, et al. (2016) Progress in physical activity over the Olympic quadrennium. Lancet 388: 1325-1336.

2. World Health Organization. Global action plan on physical activity 20182030: more active people for a healthier world. Licence: CC BY-NC-SA 3.0 IGO ed2018.

3. Ha TT TT, Harpham T, Lan PT, Thach TD, Huttly S, al. (2005) Extra Classes and Learning Outcomes of Eight-year-old Children in Vietnam. Young Lives An International Study of Childhood Poverty Working. Paper No. 29.

4. Thua Thien Hue Statistics Office (2017) Statistical Yearbook. Hue City, Thua Thien Hue Statistics Office.

5. General Statistics Office of Viet Nam: Statistical Documentation and Service Centre. 02. Population and Employment.

6. Hong TK, Trang NH, van der Ploeg HP, Hardy LL, Dibley MJ (2012) Validity and reliability of a physical activity questionnaire for Vietnamese adolescents. Int J Behav Nutr Phys Act 9: 93.

7. Hue City People's Committee. Area, population and population density in 2015 and classified by communes, wards and towns in the city.

8. Hue Citiy People's Committee. Economic Social Indicators Data.

9. World Health Organization. What is Moderate-intensity and Vigorousintensity Physical Activity?

10. Cleland CL, Hunter RF, Kee F, Cupples ME, Sallis JF, et al. (2014) Validity of the global physical activity questionnaire (GPAQ) in assessing levels and change in moderate-vigorous physical activity and sedentary behaviour. BMC Public Health. 14: 1255.

11. Boon RM, Hamlin MJ, Steel GD, Ross JJ (2010) Validation of the New Zealand Physical Activity Questionnaire (NZPAQ-LF) and the International Physical Activity Questionnaire (IPAQ-LF) with accelerometry. Br J Sports Med 44: 741-746.

12. World Health Organization (2010) Global Recommendations on Physical Activity for Health. Geneva. WHO Press. ISBN 9789241599979

13. World Health Organization (2017) Growth reference 5-19 years BMI-forage.

14. Hamari L, Kullberg T, Ruohonen J, Heinonen OJ, Díaz-Rodríguez N, Lilius J, et al. (2017) Physical activity among children: objective measurements using Fitbit One. BMC Res Notes 10: 161.

15. Verstraeten R, Lachat $C$, Ochoa-Avilés $A$, Hagströmer $M$, Huybregts $L$, et al. (2013) Predictors of validity and reliability of a physical activity record in adolescents. BMC Public Health 13: 1109.

16. Wafa SW, Aziz NN, Shahril MR, Halib H, Rahim M, et al. (2017) Measuring the Daily Activity of Lying Down, Sitting, Standing and Stepping of Obese Children Using the ActivPALTM Activity Monitor. J Trop Pediatr 63: 98-103. 